Research Article

\title{
Study of potential drug interactions between prescribed drugs in geriatric patients attending outpatient department in a government tertiary care hospital in Maharashtra
}

\author{
Tushar Bhimrao Nishandar*, Anand S. Kale, Harshal N. Pise
}

Department of Pharmacology, SRTRGMC, Ambajogai, Maharashtra, India

Received: 01 June 2016 Accepted: 01 July 2016

*Correspondence to:

Dr. Tushar Bhimrao Nishandar, Email: nishandar9696@ gmail.com

Copyright: () the author(s), publisher and licensee Medip Academy. This is an openaccess article distributed under the terms of the Creative Commons Attribution NonCommercial License, which permits unrestricted noncommercial use, distribution, and reproduction in any medium, provided the original work is properly cited.

\begin{abstract}
Background: A drug interaction is defined as a modification of the effect of a drug when it is administered with other drugs. Geriatric population is exposed to multiple drugs and consequently suffers many drug interactions (DIs). The objective of this study was to assess the potential drug interactions (PDI) in the geriatric population attending out-patient department (OPD) in a tertiary care hospital.

Methods: A cross sectional observational study was carried out from July to September 2015. Patients of either gender, age 60 years or more, attending OPD in tertiary care hospital and prescribed two or more drugs, were included in the study. Prescriptions of medical officers were screened for PDIs with Medscape drug interaction software available on the website www.medscape.com.

Results: In the present study, out of 600 prescriptions, $48.50 \%$ were identified having at least one drug interaction. Total 584 PDIs were found in $111 \mathrm{drug}$ pairs. 29.62\% PDIs were pharmacodynamic, $42.80 \%$ pharmacokinetic type and $10.78 \%$ PDIs were found affecting serum potassium level. Majority of PDIs $(61.81 \%)$ were found significant followed by minor $(36.98 \%)$ and severe $(1.19 \%)$. Ranitidine and cyanocobalamin was the most common pair showing PDI (105) followed by aspirin and enalapril (44). Aspirin was found to be the most common single drug amongst pairs to cause PDI in the present study.

Conclusions: In the present study, PDIs were studied in geriatric population. Knowledge of the prevalence and predictors of clinically important PDIs will help physicians and pharmacists identify patients at higher risk of adverse drug interactions requiring more cautious pharmacotherapy.
\end{abstract}

Keywords: Drug interaction, Geriatric, Medscape, OPD

\section{INTRODUCTION}

The drug interaction is defined as a modification of the effect of a drug when it is administered with other drug. ${ }^{1}$ The effect may be an increase or a decrease in the action of either substance, or it may be an adverse effect that is not normally associated with either drug.

Drug interactions represent a frequent cause of hospitalization, $2.8 \%$ of all hospitalizations in the elderly population. ${ }^{2}$ Drug interactions amount $6-30 \%$ of all adverse drug events. The DIs may cause significant morbidity in patients and economic burden on healthcare system. ${ }^{3}$
Aging is associated with physiological changes that may alter absorption, distribution, metabolism and excretion and response to drug. ${ }^{4}$

Geriatric population may suffer from more than one pathological condition necessitating prescription of more than one drug. ${ }^{5}$ It may be therapeutically rewarding to identify drug interactions amongst commonly prescribed drugs. A wealth of data on potential drug interactions in our elderly population may reduce morbidity and mortality in this population.

So, present study was proposed to investigate potential drug interactions between prescribed drugs in geriatric patients attending outpatient department (OPD) in a rural government tertiary care hospital in Maharashtra, India. 


\section{METHODS}

This was an observational, cross sectional study conducted in a tertiary care teaching hospital of rural Maharashtra, India. The study was conducted over a period of four months from July 2015 to October 2015 after approval from institutional ethics committee. Total of 600 patients were enrolled in the study.

\section{Inclusion criteria}

Patients of either gender, age above 60 years or more, attending OPD in tertiary care hospital and prescribed two or more drugs.

\section{Exclusion criteria}

Patients not willing to consent, patients with acute serious conditions such as myocardial infarction, diabetic coma and left ventricular failure etc.

The diagnosis and line of treatment were decided by the physician. No additional drugs or investigations were advised by us during the study period. Data of patients as per inclusion criteria was recorded. Patients were informed about the nature of research work. Written informed consent was taken from each patient before including him or her into the study.

To analyse drug interactions, software namely medscape drug interaction checker (URL- http:// reference.medscape.com/drug-interaction checker) freely available on the website www.medscape.com was used.

The clinical relevance of potential PDI was defined by a software programme namely medscape drug interaction checker, which classifies PDIs as mild, significant and serious. This software, based on clinical and pharmacological documentation, gives a reasonably fair idea of potential adverse event risk and efficacy of drugs. ${ }^{2}$

Statistical analysis was done using Microsoft Office Excel® 2007.

\section{RESULTS}

Total 600 patients were enrolled in the study. The demographic data is as shown in Table 1.

Table 1: Demographic data.

\begin{tabular}{|lll|}
\hline Gender & Number & Percentage \\
\hline Male & 366 & 61 \\
\hline Female & 234 & 39 \\
\hline
\end{tabular}

The number of drugs prescribed to all patients ranged from 2-8 per person as shown in Table 2 .
Table 2: Number of drugs per prescription.

\begin{tabular}{|lll|}
\hline $\begin{array}{l}\text { Number of drugs } \\
\text { in a prescription }\end{array}$ & $\begin{array}{l}\text { Number of } \\
\text { patients }\end{array}$ & $\begin{array}{l}\text { Total number of } \\
\text { drugs }\end{array}$ \\
\hline 2 & 131 & 262 \\
\hline 3 & 202 & 606 \\
\hline 4 & 194 & 776 \\
\hline 5 & 45 & 225 \\
\hline 6 & 23 & 138 \\
\hline 7 & 4 & 28 \\
\hline 8 & 1 & 8 \\
\hline
\end{tabular}

Potential drug interactions were detected with the help of medscape drug interaction checker. ${ }^{6}$ Out of 600 prescriptions enrolled in the study, 391 (48.50\%) were identified having at least one PDI.

Total of 584 potential drug interactions (PDI) were detected between 111 drug pairs. Ranitidine and cyanocobalamin was the most common pair showing PDI (105) followed by aspirin and enalapril (44).

Medscape drug interaction software identified potential PDIs into three categories- minor, significant and serious. Out of 584 PPDIs, $7(1.19 \%)$ serious, $361(61.81 \%)$ significant while $216(36.98 \%)$ were minor.

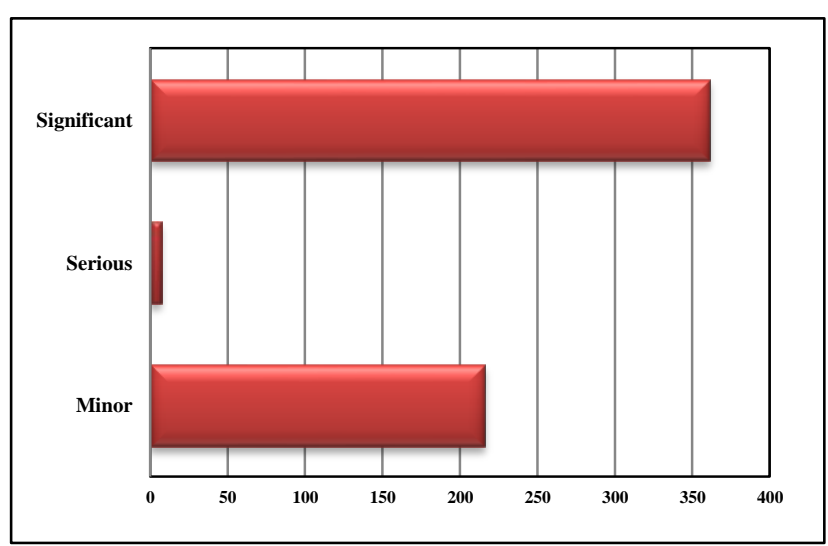

Figure 1: Drug interactions as per their severity.

Serious interactions were detected between ibuprofen and aspirin (3), ciprofloxacin and ondensetron (2), telmisartan and enalapril (1) and rabeprazol and digoxin (1).

Table 3: Severe interactions.

\begin{tabular}{|ll|}
\hline Drug Pair & $\begin{array}{l}\text { Number of times of } \\
\text { interaction }\end{array}$ \\
\hline Ibuprofen + aspirin & 3 \\
\hline Telmisartan + enalapril & 1 \\
\hline Rabeprazole + digoxin & 1 \\
\hline Ciprofloxacin + ondansetron & 2 \\
\hline
\end{tabular}

Medscape drug interaction checker software detected out of 584 PDIs causally $42.8 \%$ were pharmacokinetic, 
$29.62 \%$ pharmacodynamic, in $16.78 \%$ the mechanism was unknown and $10.78 \%$ were found to affect serum potassium level.

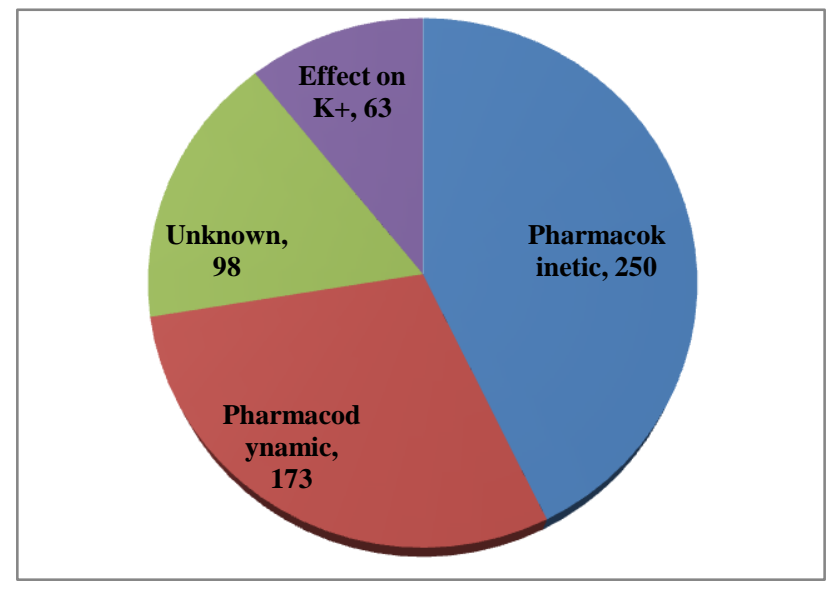

Figure 2: Mechanisms of PDIs.

Out of 250 pharmacokinetic PDIs, 149 were known to affect absorption, 51 Excretion, 32 Metabolism and 12 Distribution.



Figure 3: Distribution of pharmacokinetic PDIs.

Total of 173 pharmacodynamic PDIs were identified. 95 were synergistic and 78 were antagonistic.

In present study, aspirin was the most common drug with potential of interactions. 146 PDIs were attributed to it, followed by amlodipine (131), enalapril (109), cyanocobalamin (108) and atenolol (96).

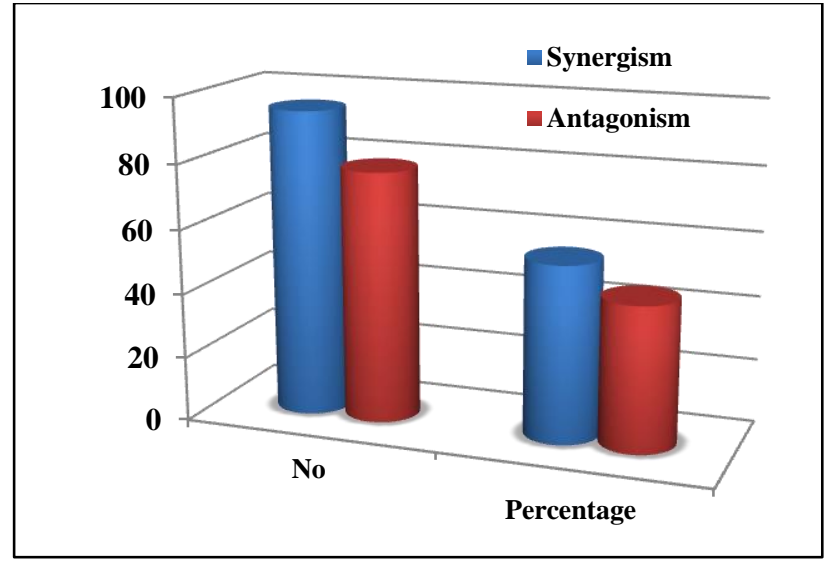

Figure 4: Distribution of pharmacodynamic interactions.

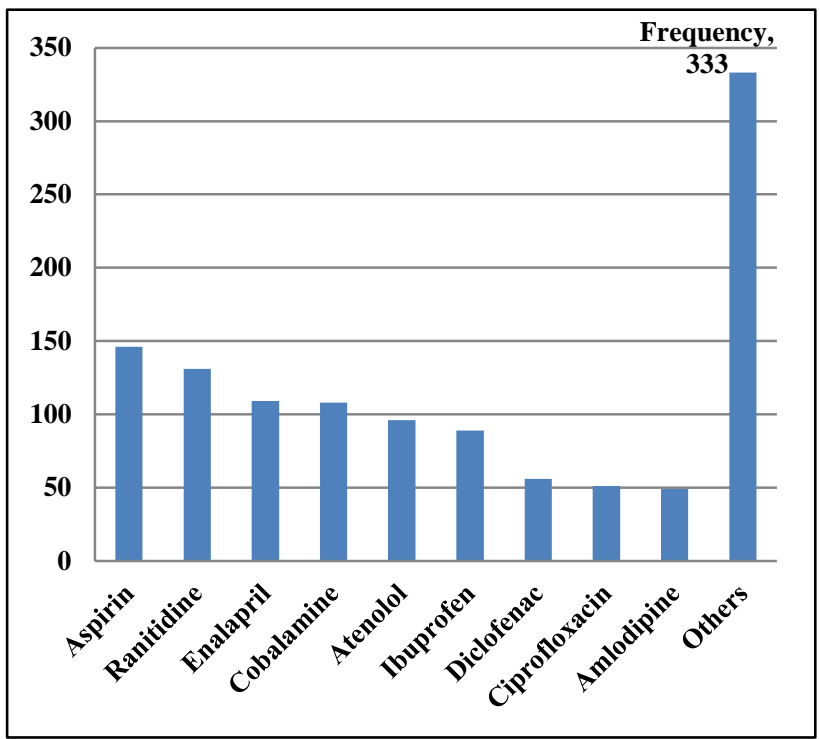

Figure 5: Common drugs involved in drug interactions.

\section{DISCUSSION}

Drug interaction refers to modification of response to one drug by another when they are administered simultaneously or in quick succession. The modification is mostly quantitative, i.e. the response is either increased or decreased in intensity. ${ }^{7}$

Adverse drug reactions are significant cause hospital of admission. Many of the adverse drug reactions are caused by drug interactions. ${ }^{8}$ Elderly people are more susceptible for drug interactions owing to poly-pharmacy. Some PDI are common and elderly patients should be monitored for them. The data regarding PDI in elderly patients in India is scarce. Therefore, this study was planned.

In this study potential drug interactions were detected using medscape drug interaction checker software. It is a web portal from WebMD which is a part of WebMD health professional network. It is a reputed online portal. 
Many other softwares have been used in various studies which include Micromedex drug interaction checker, Drugs.com/drug interaction checker etc.

A total of 584 PDIs were identified in 600 patients involving 78 different drugs with a total of 111 different drug combinations. In this study, the prevalence of PDI was found to be $48.50 \%$ which is comparable to $41.5 \%$ in the study of Bjorkman et al Kothari et al studied PDIs in patients of hypertension and reported overall incidence of $71.5 \% .^{2,9}$ This is different on the account of differing inclusion criteria.

The possibility of drug interaction rises whenever patient concurrently receives more number of drugs. In the present study, patients were prescribed on an average 3.41 drugs. Many of the commonly used drugs in geriatric patients interact with each other. These drugs can be used together to treat various conditions following a riskbenefit assessment. It is desirable that many clinicians balance the risk of PDIs against benefits while prescribing drugs to the geriatric patients.

Of the total identified PDIs $61.8 \%$ were of significant severity. Causally $42.6 \%$ were pharmacokinetic, $29.68 \%$ pharmacodynamic, in $16.78 \%$ the basis of drug interaction was unknown and in $10.78 \%$ drug interactions affected serum potassium level. These findings are comparable to $76 \%$ Pharmacokinetic and $22 \%$ Pharmacodynamic interactions in the study of Vonbach and Aparasu. ${ }^{10,11}$

Most common drug involved in PDI is Aspirin, an antiplatelet drug followed by amlodipine, a calcium channel blocker and enalapril, an ACE inhibitor. Aspirin was involved in $25 \%$ (146) of PDIs, which is lesser than the reported incidence by Patel VK et al. (44.84\%) which may be attributed to the selection of patients of heart disease only. ${ }^{12}$

In this study, significant number of patients was at risk of developing potential drug interaction. Proper management of PDIs is based on recognition of the PDIs and consequently taking the suitable measures like therapeutic drug monitoring and dose adjustment, inclusion of corrective agents to reduce the likelihood of an adverse outcome eg. omeprazole for gastric protection.

Despite the great heterogeneity in health status and functional levels within the elderly population, aging generally increases an individual's risk of illness and, subsequently use of medications. The frequent presence of coexisting illnesses and the use of multiple medications by the elderly increase the potential for drug interactions. ${ }^{4}$ In such scenario of increased risk of interactions and potential adverse outcomes, there is a need to establish database of PDIs in our patients, to monitor them and to take suitable preventive or corrective measures.

\section{Limitations of this study}

There are some limitations of this study. We have collected data from only one institute, therefore population is relatively homogenous. The utilization of PDI-checker software provides only a 'potential' estimate of occurrence of PDI. This approach does not take into account the actual occurrence of adverse effects. However, despite these limitations, this approach is currently widely used to assess the clinical relevance and risk of exposure to.

\section{CONCLUSION}

This study shows the overall incidence of PDIs was $48.50 \%$. Majority interactions are of significant severity. Most of the interactions are pharmacokinetics type, most of them due to changes in absorptive mechanism. Aspirin is the most commonly involved drug in interactions and hence needs intensive monitoring during therapy.

Knowledge of the prevalence and predictors of clinically important PDIs will help clinicians and pharmacists to identify patients at higher risk of PDI-related adverse drug reactions, which require cautious use of medications to avoid adverse outcome.

More studies from various medical centres are needed to establish data on PDIs (potential drug interactions) in geriatric population.

\section{Funding: No funding sources}

Conflict of interest: None declared

Ethical approval: Institutional Ethics Committee, SRTR

GMC, Ambajogai. Dist. Beed. Letter No. Outward No/SRTRGMC/PHAR/IEC/211/2015 dated 27/07/2015

\section{REFERENCES}

1. Patel PS, Rana DA, Suthar JV, Malhotra SD, Patel VJ. A study of potential adverse drug-drug interactions among prescribed drugs in medicine outpatient department of a tertiary care teaching hospital. J basic Clin Pharm. 2014;5(2):44-8.

2. Kothari N, Ganguly B. Potential drug-drug interactions among medications prescribed to hypertensive patients. J Clin Diagnostic Res. 2014;8(11):1-5.

3. Chavda NB, Solanky PP, Baria H, Naik R, Bharti K. Study of potential drug-drug interaction between prescribed drugs in patients attending outpatient department of medicine at tertiary-care hospital in south Gujarat region. Natl J Physiol Pharm Pharmacol. 2015;5(3):236-42.

4. Interactions D, Issues S, Populations EA, Committee WS, Interaction D, Isbn M, et al. Pharmacokinetics and drug interactions in the elderly and special issues in elderly African- American Populations. 1997. 
5. Costa AJ. Potential drug interactions in an ambulatory geriatric population. Fam Pract. 1991;8(3):234-6.

6. Website Available

at http://reference.medscape.com/druginteractionchecker. Accessed on 1 June 2016.

7. Tripathi KD. Drug interaction. Essentials of medical pharmacology. $7^{\text {th }}$ ed. New Delhi: Jaypee; 2013:92834.

8. Beard K. Adverse reactions as a cause of hospital admission in the aged. Drugs aging. 1992;2:356-67.

9. Bjorkman IK, Fastbom J, Schmidt IK, Bernsten CB. The pharmaceutical care of the elderly in Europe research (PEER) group. Drug-drug interactions in the elderly. Ann Pharmacother. 2002;36:1675-81.
10. Aparasu R, Baer R, Aparasu A. Clinically important potential drug-drug interactions in outpatient settings. Research in social and administrative pharmacy. 2007;3:426-37.

11. Vonbach P, Dubied A, Krahenbuhl S, Beer JH. Prevalence of drug-drug interaction at hospital entry and during hospital stay of patients in internal medicine. Eur J Int Med. 2008;19:413-20.

12. Patel VK, Acharya LD, Rajakannan T, Mallayasamy S, Guddttu V, Padmakumar R. Potential drug interactions in patients admitted to cardiology wards of a south Indian teaching hospital. AMJ. 2011;4(1):9-14.

Cite this article as: Nishandar TB, Kale AS, Pise HN. Study of potential drug interactions between prescribed drugs in geriatric patients attending outpatient department in a government tertiary care hospital in Maharashtra. Int J Basic Clin Pharmacol 2016;5:1569-73. 